Case Report

\title{
Dialysis Dependent 'Double Positive Disease' Responding To Immunosuppression : A Case Report
}

\author{
Shashidhar Baikunje ${ }^{1}$, Mahesha Vankalakunti ${ }^{2}$, Adithi Bhandary ${ }^{3} \&$ P. S. Prakash ${ }^{3}$ \\ ${ }^{1}$ Associate Professor \& HOD, Department of Nephrology, ${ }^{3}$ Senior Resident, ${ }^{4}$ Professor and HOD, Department of Medicine, \\ K. S. Hedge M edical Academy, Nitte University, M angalore, \\ ${ }^{2}$ Consultant Renal Histopathologist, M anipal Hospital, Bengaluru, Karnataka, India. \\ *Corresponding Author : Shashidhar Baikunje, Associate Professor \& HOD, Department of Nephrology, K. S. Hegde M edical Academy, \\ Mangalore 575018, Karnataka, India. \\ Mobile: +919686142218 E-mail : baikunje@ hotmail.com
}

$\begin{array}{ll}\text { Received } & : 08-06-2015 \\ \text { Review Completed } & : \text { 28-03-2016 } \\ \text { Accepted } & : 05-05-2016\end{array}$

Keywords : anti-GBM disease, plasma exchange, vasculitis.

\begin{tabular}{|c|}
\hline Access this article online \\
\hline Quick Response Code \\
\hline
\end{tabular}

\begin{abstract}
:
The outcome of patients with crescentic nephritis and the presence of both ANCA and anti GBM disease (double positive disease) is controversial. Initial data supported the view that this condition has a more favorable prognosis than anti GBM disease but larger and more recent series found much worse renal outcome especially in patients with severe renal failure requiring dialysis. We present a case with severe renal failure due to double positive disease who recovered renal function with aggressive immunosuppression including steroids, cyclophosphamide and plasma exchange.
\end{abstract}

\section{Introduction}

The clinical significance of combined ANCA and anti-GBM antibodies is unclear and the relationship between the two antibodies is unknown. In the appropriate clinical setting, when both the antibodies are present, the condition is called double positive disease. In double positive disease renal involvement is very common and often severe. For example, in a large series, renal disease was present in hundred percent and seventy percent required dialysis within 48 hours of presentation ${ }^{1}$.

\section{Case History}

A 48 year old Indian lady with generalized arthralgia and malaise since one year was admitted with fever, oliguria, oedema, tiredness and shortness of breath of two weeks duration. She had acute kidney injury with a creatinine of $7.2 \mathrm{mg} / \mathrm{dL}$ Urine analysis showed microhaematuria and significant proteinuria. She had normocytic normochromic anaemia with a haemoglobin of 8.7 $\mathrm{gm} / \mathrm{dl}$, neutrophilicleucocytosis with white cell count of 15700/cu.mm, normal platelet count and raised inflammatory markers. Coagulation profile was normal. Blood and urine cultures were negative. She had normal sized kidneys on ultrasound. Chest x-ray showed mild congestion. PR3-ANCA was strongly positive at $89.99 \mathrm{U} / \mathrm{ml}$. She had hemodialysis through internal jugular catheter. A provisional diagnosis of ANCA associated vasculitis was made. A renal biopsy was carried out which is shown below (Figure $1 \& 2$ ).

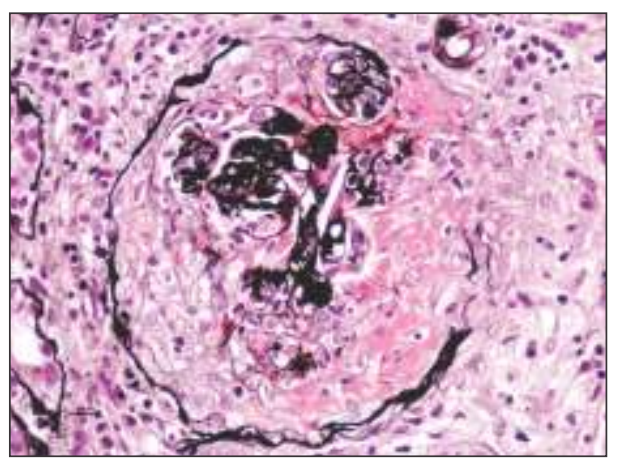

Figure 1 : Glomerulus possessing circumferential active cellular crescent with necrotizing lesion; rupture of capillary basement membranesand Bowman's capsule (40x, PASM stain). 


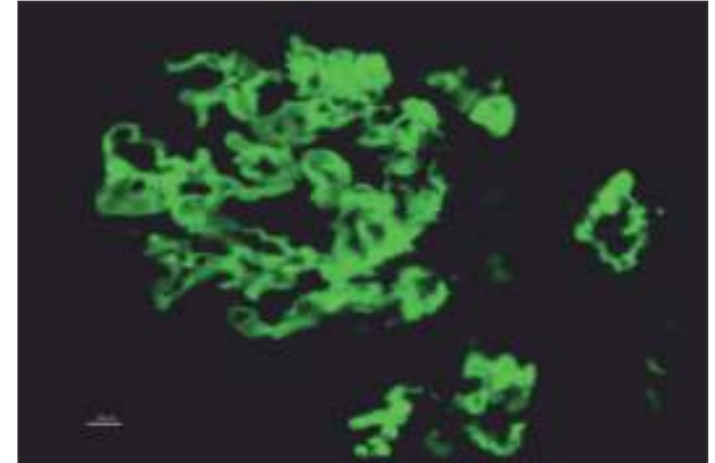

Figure 2 : Glomerulus revealing linear pattern of deposits along the capillary basement membranes with IgG ( $3+$ on a scale of 0 to $4+)$.

The history, clinical and laboratory findings were compatible with vasculitis. Strongly positive PR3 ANCA and biopsy findings suggestive of crescentic glomerulonephritis (nine out of twelve glomeruli showed active cellular crescents) supported the diagnosis of ANCA associated vasculitis. But the immunoflourescence showed linear pattern of deposits along the capillary basement membrane with $\lg G$ and this is considered pathognomonic of anti GBM disease. Anti GBM antibody was tested subsequently and it was strongly positive. Even though more recent evidence suggests that patients with doublepositive disease who are dialysis dependent tend to have bad renal prognosis in spite of aggressive immunosuppression including plasma exchange, we decided to treat our patient with methylprednisolone followed by oral prednisolone, IV cyclophosphamide and plasma exchange ( 6 exchanges of 2.5 litres on alternate days) and the patient made remarkable recovery. Her renal function gradually improved, dialysis was discontinued on day 16 and the creatinine settled down to $1.3 \mathrm{mg} / \mathrm{dL}$ over the next few weeks (Table 1).

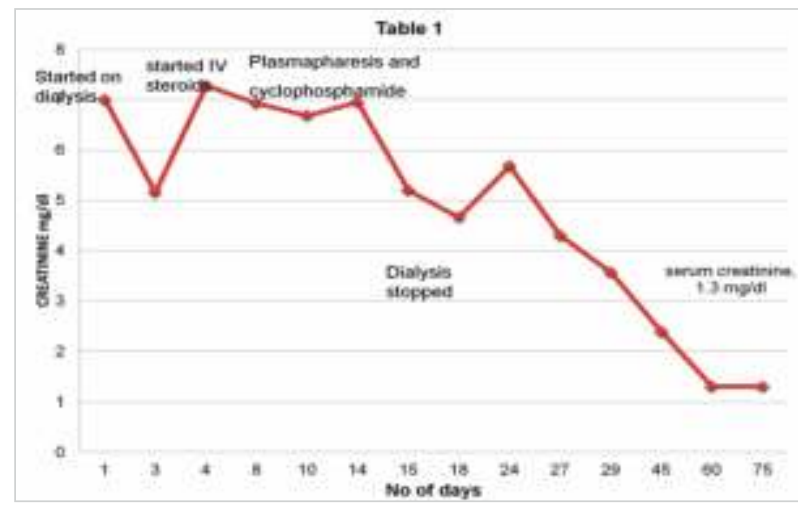

\section{Discussion}

Systemic symptoms and signs, such as malaise, fever, weight loss or arthralgia are typically absent in anti GBM disease. The presence of such features in a patient with biopsy picture compatible with anti GBM disease should prompt one to consider double positive disease as in our patient who had malaise and generalised arthralgia since a year.

Double positive disease was previously thought to have better renal prognosis than anti-GBM diseasewith as many as 40 percent of such patients recovering some renal function ${ }^{2}$. However larger and more recent studies found much worse renal outcome ${ }^{1,3}$. In one of those studies, among double-positive patients who were dialysis dependent at presentation, none of them recovered renal function in spite of immunosuppression with or without plasma exchange 1 . Other study concluded that the renal survival in double positive patients is similar to patients with anti-GBM disease and worse compared to patients with ANCA associated vasculitis ${ }^{3}$. Our case supports the previously held view that the prognosis may be better than patients with anti GBM disease and makes a case for aggressive immunosuppression including plasma exchange in such patients.

The immunoflourescence showing linear pattern of deposits along the capillary basement membrane with IgG is considered pathognomonic of anti GBM disease but linear IgG on immunoflorescence can also be seen in diabetic nephropathy and fibrillary glomerulonephritis ${ }^{4}$. But the staining is usually not very strong and it is quite coarse with fibrillary glomerulonephritis. These two conditions can be easily distinguished from anti-GBM disease or "double-positive" disease based on the clinical presentation and histology.

In conclusion, double positive disease has variable prognosis and it is worth considering aggressive immunosuppression including plasma exchange in suitable patients with double positive disease irrespective of the severity of renal involvement at presentation.

Funding-None 


\section{References}

1. Levy JB, Hammad T, Coulthart A, Dougan T, Pusey CD. Clinical features and outcome of patients with both ANCA and anti-GBM antibodies. Kidney Int. 2004; 66(4):1535.

2. Jayne DR, Marshall PD, Jones SJ, Lockwood CM. Autoantibodies to GBM and neutrophil cytoplasm in rapidly progressive glomerulonephritis.Kidney Int. 1990; 37(3):965.

3. Rutgers $A$, Slot $M$, van Paassen $P$, van Breda Vriesman $P$, Heeringa $P$, Tervaert JW. Coexistence of anti-glomerular basement membrane antibodies and myeloperoxidase-ANCAs in crescentic glomerulonephritis. AmJ Kidney Dis. 2005; 46(2):253.

4. Alpers CE, Rennke HG, Hopper J Jr, Biava CG. Fibrillary glomerulonephritis: an entity with unusual immunofluorescence features. Kidney Int. 1987; 3 